\title{
Capacidade funcional, condições socioeconômicas e de saúde de idosos atendidos por equipes de Saúde da Família de Goiânia (GO, Brasil)
}

\author{
Functional capacity, socioeconomic conditions and of health \\ of elderly assisted by Family Health teams in Goiania (GO, Brazil)
}

Daniella Pires N unes ${ }^{1}$

Adélia Yaeko Kyosen Nakatani ${ }^{1}$

Érika Aparecida Silveira ${ }^{2,3}$

M aria M árcia Bachion ${ }^{1}$

M arta Rovery de Souza ${ }^{3,4}$

${ }^{1}$ FaculdadedeEnfermagem, UniversidadeFederal de Goiás. Rua $227 \mathrm{~s} / \mathrm{n}$, quadra 68, Setor Leste

Universitário. 74605-080 Goiânia GO.

dpiresnunes@yahoo.com.br ${ }^{2}$ Faculdade deN utrição, UniversidadeFederal de Goiás.

${ }^{3} \mathrm{~N}$ úcleo deEstudosem Saúde Coletiva, UniversidadeFederal de Goiás.

${ }^{4}$ FaculdadedeFilosofiae

Ciências Humanas,

UniversidadeFederal de

Goiás.
Abstract The population ageing is a challenge for health professionals. This study seek to evaluate the functional capacity and to identify the factors associated to the dependence for the activities of daily life (ADL) and activities instrumental of daily life (AIDL), and also to describe the socioeconomic, demographic and health profile of the elderly. A transverse study was carried out with seniors assisted by Family $\mathrm{H}$ ealth teams of the District East Sanitary of Goiania (GO). A questionnairewas applied to obtain socioeconomic and demographic date, factors related to the health and scales of the functional evaluation. For analyses it was used Square Qui Test considering the level of significance of $5 \%$. The sample wascomposed by 388 elderly, $57,5 \%$ with age varying from 60 to 69 years, 58,5\% female, 39,7\% illiterate, $77,3 \%$ with income $\leq 2$ minimum wages and $70,9 \%$ bearers of chronic diseases. It was verified that $34,8 \%$ needed help in one or moreADL and $60,6 \%$ in AIDL. The factors more frequently associated at dependence in both activities were: balanceand mobility debi litated, depression, cognitive deficit and age $\geq 80$ years. These results denote that the elderly are living aging characterized by comorbidities and dependence to accomplish daily activities.

Key words Aged, Family Health, Daily activities, Independence, Autonomy
Resumo 0 envelhecimento populacional é um desafio para os profissionais de saúde. Este estudo objetivou avaliar a capacidade funcional e identificar os fatores associados à dependência para as atividades de vida diária (AVD) e instrumentais de vida diária (AIVD) e descrever o perfil socioeconômico, demográfico e de saúde de idosos. Trata-se de pesquisa transversal realizada com idosos atendidos pelas equipes de Saúde da Família do Distrito Sanitário Leste de Goiânia (GO). A plicou-se questionário para obtenção de dados socioeconômicos e demográficos, fatores relacionados à saúdee escalas de avaliação funcional. Para análise, utilizou-se o teste Qui-Quadrado, conside rando o nível de significância de 5\%. A amostra foi composta por 388 idosos, sendo $57,5 \%$ entre 60 e 69 anos, $58,5 \%$ do sexo feminino, 39,7\% analfabetos, $77,3 \%$ com renda $\leq 2$ salários mínimos; $70,9 \%$ referiram doenças crônicas. Verificou-se que $34,8 \%$ necessitavam deajuda em uma ou mais AVD e $60,6 \%$ nas AIVD. Os fatores mais frequentemente associados a essa dependência em ambas as atividades foram: equilíbrio e mobilidade prejudicados, depressão, déficit cognitivo e idade $\geq$ 80 anos. Esses resultados denotam que os idosos estão vivenciando envelhecimento caracterizado por comorbidades e dependência para realizar as atividades diárias.

Palavras-chave Idosos, Saúde da Família, Atividades cotidianas, Independência, Autonomia 
Introdução

O envelhecimento populacional éconsiderado um dos maiores desafios da Saúde Pública, principalmente nos países em desenvolvimento. Esse processo acontece de forma rápida, sem tempo para uma reorganização social e da área de saúde mais adequadas para atender às novas demandas ${ }^{1-3}$.

Estudos têm revelado que a maioria dos idosos ainda possui renda igual ou inferior a um salário mínimo e gasta aproximadamente um quarto da renda com medicamentos, interferindo assim na sua qualidade da vida ${ }^{4-5}$. As condições de saúde da população idosa também podem ser determinadas pel os perfis de morbidade e de mortalidade, presença de déficits físicos e cognitivos e frequência na utilização de serviços desaúde. A investigação da capacidadefuncional é um dos grandes marcadores da saúde do idoso, e vem emergindo como componente-chave para a avaliação da saúde dessa população $0^{5-6}$.

Veras ${ }^{7}$ refere que a manuten ção da capacidade funcional deve ser interligada com a autonomia, a independência física emental. A independência e a autonomia podem ser determinadas por meio das atividades de vida diária (AVD) e das atividades instrumentais de vida diária (AIVD). As atividades de vida diária são as relacionadas ao autocuidado, e as atividades instrumentais de vida diária estão relacionadas com a participação do idoso em seu ambiente social e indicam a capacidade do indi víduo de levar uma vida independente, dentro da comunidade ${ }^{2,8}$. A dificuldadeou incapacidade do idoso em realizar tais atividades associa-se ao aumento do risco de mortalidade, hospitalização, necessidade de cuidados prolongados e elevado custo para os serviços de saúde 9 .

A Estratégia de Saúde da Família (ESF), implantada no Brasil em 1994 e em Goiânia a partir de 1998, visa à reorganização da Aten ção Básica no País, de acordo com os preceitos do Sistema Único de Saúde (SUS) ${ }^{10}$. Em 2006, pactuou-sena Atenção Básica a implantação do Programa Saúde do I doso. Diante disso, os profissionais envolvidos na atenção devem cumprir a Política Nacional do Idoso e o Estatuto do I doso, garantindo a ele um envelhecimento saudável edigno ${ }^{11}$.

Trata-se de uma estratégia recente. $\mathrm{N}$ as unidades de saúde, os idosos têm sido acompanhados apenas por meio dos programas de hipertensão e diabetes (Hiperdia) estabelecidos pelo M inistério da Saúde, junto com os demais segmentos de idade adulta. Outros idosos são atendidos no contexto da demanda espontânea nas unidades. As equipes da Saúde da Família ainda não investiram em inquéritos populacionaispara identificar a situação de saúde dos idosos; assim, carecem deinformações sistematizadas sobre esse grupo.

O presente estudo teve como objetivos: avaliar a capacidade funcional, identificar os fatores associados à dependência e descrever os perfis socioeconômico, demográfico edesaúdedosidososatendidos por equipes da Saúde da Família do Distrito Sanitário Leste do município de Goiânia (GO).

Os resultados obtidos poderão oferecer subsídios para redirecionamento das práticas dos profissionais de saúde, no sentido da integralidade do atendimento. 0 conhecimento produzido poderá oferecer novos subsídios para a formação dos profissionais de saúde, com vistas à avaliação de saúde ampliada.

\section{Métodos}

Trata-se de um estudo transversal realizado com idosos (idade $\geq 60$ anos) atendidos por equipes da Estratégia de Saúde da Família do Distrito Sanitário Leste (DSL) do município de Goiânia (GO). Elegeu-se o DSL por ser uma região de atuação da UniversidadeFederal deGoiás (UFG) na Atenção Básica.

A população estimada para o ano de 2006 , no município de Goiânia, foi de 1.225.410 habitantes; destes, aproximadamente $7 \%$ representaram pessoas com 60 ou mais anos de idade 3 . 0 município conta com 11 Distritos Sanitários, e o DSL possui 120.216 habitantes, sendo $6,9 \%$ deidosos $^{10}$. Neste distrito, a maioria das moradias é de alvenaria, com $100 \%$ de energia elétrica, abastecimento deágua tratada, coleta de lixo adequada, equase toda a área é beneficiada com sistema de esgoto sanitário público. A região fica distante aproximadamente dez quilômetros do centro da cidade, sendo bem servida por transporte coletivo.

Para o cálculo do tamanho da amostra, foram considerados dois desfechos e duas variáveis de exposições de interesse principal: dependência paraAVD (35\%) edependência para AIVD (60\%), depressão ealteração de equilíbrio emobilidade, respectivamente. Os parâmetros utilizados para o tamanho da amostra foram: nível de confiança de $95 \%$, poder de $80 \%$, razão de não expostos:expostos de 3:1, razão de prevalência de 2 , prevalência da doença entre os não expostos $18 \%$ e nos expostos de $36 \%$. Assim, o tamanho da amostra foi estimado em 268 idosos. Considerando-se o acréscimo de $10 \%$ para even- 
tuais perdas e recusas e de $30 \%$ para controle de fator de confusão e estratificação, a amostra necessária incluiu 375 indivíduos.

O DSL possui 12 equipes de Saúde da Família ${ }^{10}$; destas, procedeu-se ao sorteio de seis equipes da ESF. Foram convidados para participarem da pesquisa os idosos residentes nas áreas de abrangência dessas seis equipes.

Cada equipe solicitou ao agente comunitário a listagem dos idosos cadastrados e respectivos endereços. A coleta de dados foi realizada no período de novembro de 2001 a dezembro de 2005 , nos meses de novembro, dezembro, janei ro efevereiro, de acordo com a disponibilidadeda equipe de pesquisadores de campo.

Idosos em situação de comprometimento da condição de saúde, com dificuldade de expressão, podem ser vistos como pessoas em situação devulnerabilidade, do ponto devista da bioética, com autodeterminação reduzida, e desta forma com capacidade afetada para o autêntico consentimento livree esclarecido. No entanto, os instrumentos de coleta dedadosincluíam escalas de autoavaliação, que requeriam respostas relativas à autopercepção. Assim, para os propósitos desteestudo, consideraram-se os seguintes crité rios de inclusão: ter idade igual a/ou maior de 60 anos, residir na área de abrangência das equipes selecionadas e ter condições de responder à entrevista sem ajuda do cuidador.

Outrosinstrumentos deavaliação incluíam a aval iação deatividades que dependem da capacidade de deambulação. Assim, adotou-se como critério de exclusão: estar acamado e retirar o consentimento a qualquer tempo de sua participação no estudo.

0 instrumento de coleta de dados foi um questionário padronizado aplicado por acadêmicos de enfermagem, após capacitação prévia pelo pesquisador-coordenador. Para a coleta de dados, os acadêmicos visitavam o domicílio do idoso acompanhado pelo agente comunitário de saúde (ACS) para facilitar a abordagem pesquisador-pesquisado. 0 entrevistador esclarecia que sua participação não causaria nenhum dano físico ou moral equeseriam garantidoso sigilo eo anonimato de suas informações, caso o idoso concordasse com a entrevista.

A entrevista foi realizada mediante aplicação do questionário e escalas que abordavam dados socioeconômicos e demográficos (idade, sexo, estado civil, religião, profissão, escolaridade, renda, tipo de moradia), fatores relacionados à saúde (quedas sofridas recentemente, acuidade visual, auditiva, tabagismo, etilismo e morbidades referidas). As morbidades referidas investigadas foram: hipertensão arterial, artrite, artrose ou reumatismo, diabetes, osteoporose, problemas cardíacos, doença crônica pulmonar, câncer e acidente vascular cerebral.

Foram aplicadas em todos os idosos as seguintes escalas de avaliação funcional: (1) miniexame do Estado M ental de Folstein \& Folstein, para a avaliação cognitiva ${ }^{12}$; (2) escala de depressão geriátrica de Yesavage, para as condições emocionais do idoso, na versão simplificada, validada por Almeida e Almeida13; (3) escala de Barthel, para mensurar as atividades de vida diária14; (4) escala de Lawton, para exame das atividades instrumentais de vida diária ${ }^{15}$.

Para que não houvesse interferência do grau de escolarização do miniexame do estado mental de Folstein \& Folstein, consideraram-se os escores de 19 como ponto de corte para analfabetos, 23 para aqueles que possuíam de um a três anos de escolaridade, 24 para quatro a sete anos e 28 para aqueles com mais de sete anos de escolaridade, conforme descreve a metodologia descrita por Laks et al. ${ }^{16}$.

Utilizou-se o programa Epi-info 6.0 para estruturação do banco de dados e análise estatística. Para verificar a associação entre as variáveis estudadas, aplicaram-se o Qui-quadrado de Pearson e o Teste Exato de Fisher, considerando como nível de significância valor de $p \leq 0,05$. A estimativa de efeito foi avaliada pelo cálculo da razão de prevalência, com intervalo de confiança de $95 \%$.

$\mathrm{Na}$ avaliação de cada uma das AVD e AIVD, foi considerada dependência parcial quando 0 idoso relatava precisar de algum tipo de suporte ou apoio, porém participava ativamente; e dependência total quando o idoso relatava ser incapaz de realizar a atividade, necessitan do de ajuda, com participação mínima ou nenhuma.

O julgamento global de independência, dependência parcial ou total foi realizado mediante análise dos escores totais obtidos em cada uma das escalas. Para avaliação de AVD, é considerado independente 0 idoso que alcança 100 pontos; para dependência parcial, o idoso deve perfazer entre 70 e 95 pontos. A dependência total é caracterizada quando o idoso apresenta menos que 70 pontos ${ }^{14}$.

Em relação às AIVD, é considerado estado de independência quando o idoso apresenta 24 pontos e dependência parcial entre 9 e 23 pontos. A dependência total é evidenciada quando o idoso apresenta 8 pontos ${ }^{15}$.

Considerou-se como desfecho a situação de dependência, agrupando-se os estados de de- 
pendências parcial e total. Nesse sentido, realizou-se análise bivariada de dependência para AVD e AIVD com variáveis sobre condições de saúde, sexo eidade. As variáveis estatisticamenteassociadas foram posteriormente estratificadas para cada tipo de AVD (alimentar-se, banhar-se, vestir-se, usar sanitário, deambular, passar de cama para cadeira, subir e descer escadas, mover-sena cama e continência urinária efecal) eAIVD (preparar alimentos, realizar tarefas domésticas, lavar roupas, manusear medicamentos, usar telefone, manusear tel efone, fazer compras, utilizar meio de transporte).

O projeto foi aprovado pelo Comitê de Ética em Pesquisa (COEP), da Universidade Federal de Goiás, com protocolo no 26/2001. Todos os idosos receberam e assinaram o Termo de Consentimento Livree Esclarecido ${ }^{17}$.

\section{Resultados}

Foram convidados a participar da pesquisa 407 idosos. Destes, $11(2,7 \%)$ não preencheram os critérios de inclusão e oito $(1,96 \%)$ recusaram. Participaram do estudo 388 idosos, sendo que $30,9 \%$ procediam de Goiânia einterior de Goiás. Entre os demais, 23,6\% eram da Bahia, 20,0\% de Minas Gerais, 7,4\% do Tocantins e $18,1 \%$ de outros estados.

$\mathrm{Na}$ Tabela 1, pode ser vista a distribuição das características socioeconômicas e demográficas. Observou-seque $58,5 \%$ dos idosos eram do sexo feminino, 57,5\% tinham entre60 e 69 anos, 50,8\% eram casados, $57,5 \%$ católicos, $43,8 \%$ tinham de um a cinco anos de escolaridade, $77,3 \%$ apresentavam rendimento $\leq 2$ salários mínimos e 88,7\% possuíam moradia própria.

A maioria dos entrevistados (59,8\%) morava em domicílios multigeracionais, apresentando média aproximada de quatro pessoas por domicílio. $N$ ão desempenhavam atividades profissionais na época da avaliação $82,7 \%$, e destes, $70,4 \%$ eram aposentados. Dos que trabalhavam $(17,3 \%)$, a maioria realizava serviços gerais $(64,8 \%)$; os demais eram autônomos (15,5\%), realizavam serviços domésticos $(12,7 \%)$ ou eram comerciantes (7\%).

Quanto às condições de saúde descritas na Tabela 2, nota-se que $68,3 \%$ possuíam acuidade visual comprometida, 35,1\% manifestaram escores sugestivos para depressão, 23,7\% apresentaram equilíbrio e mobilidade prejudicados, $38,7 \%$ referiram quedas, $70,9 \%$ relataram ter uma ou mais doenças, principalmentehipertensão arterial (51\%).
Em relação à capacidade do idoso em executar as AVD, distingue-se na Tabela 2 que 31,7\% eram parcialmentedependentes $\mathrm{e}, 1 \%$ totalmente dependentes. Concernente às AIVD, de acordo com o relato dos participantes, $45,7 \%$ apresentavam dependência parcial e 14,9\% dependência total.

As AVD mais comprometidas, conforme pode ser observado no Gráfico 1, que geraram dependências (parcial e total, respectivamente), foram: incontinência urinária $(20,9 \% ; 1,3 \%)$ e subir e descer escadas (12,6\%; 3,1\%). As princi-

Tabela 1. Características socioeconômicas e demográficas dos idosos atendidos por equipes de Saúde da Família do Distrito Sanitário Leste. Goiânia (GO), 2001-2005 (n=388).

\begin{tabular}{|c|c|c|}
\hline Características & $\mathrm{n}$ & $\%$ \\
\hline \multicolumn{3}{|l|}{ Sexo } \\
\hline Feminino & 227 & 58,5 \\
\hline M asculino & 161 & 41,5 \\
\hline \multicolumn{3}{|l|}{ Faixa etária } \\
\hline $60-69$ anos & 223 & 57,5 \\
\hline $70-79$ anos & 119 & 30,7 \\
\hline 80 ou mais anos & 46 & 11,8 \\
\hline \multicolumn{3}{|l|}{ Estado civil } \\
\hline Casado & 197 & 50,8 \\
\hline Viúvo & 131 & 33,8 \\
\hline Desquitado-divorciado & 41 & 10,5 \\
\hline Solteiro & 19 & 4,9 \\
\hline \multicolumn{3}{|l|}{ Religião } \\
\hline Católica & 223 & 57,5 \\
\hline Evangélica & 135 & 34,8 \\
\hline Sem religião & 16 & 4,1 \\
\hline Espírita & 14 & 3,6 \\
\hline \multicolumn{3}{|l|}{ Escolaridade } \\
\hline Sem instrução & 154 & 39,7 \\
\hline$<1$ ano & 34 & 8,8 \\
\hline $1-5$ anos & 170 & 43,8 \\
\hline 6 ou mais anos & 30 & 7,7 \\
\hline \multicolumn{3}{|l|}{ Renda } \\
\hline Sem renda & 34 & 8,8 \\
\hline <2 salários mínimos & 300 & 77,3 \\
\hline > 2 salários mínimos & 54 & 13,9 \\
\hline \multicolumn{3}{|l|}{ Moradia } \\
\hline Própria & 344 & 88,7 \\
\hline Alugada & 27 & 7,0 \\
\hline Cedida/outros & 17 & 4,3 \\
\hline \multicolumn{3}{|l|}{ Quantidade de moradores } \\
\hline Até 2 pessoas & 156 & 40,2 \\
\hline 3 a 4 pessoas & 137 & 35,3 \\
\hline 5 a 6 pessoas & 61 & 15,7 \\
\hline M ais de 6 pessoas & 34 & 8,8 \\
\hline
\end{tabular}


pais AIVD comprometidas foram: utilizar meio detransporte $(27,1 \% ; 8,0 \%)$, realizar tarefas domésticas (23,2\%; $10,8 \%)$, usar telefone $(21,4 \%$; $10,1 \%$ ) e lavar roupas $(20,6 \% ; 14,9 \%)$, para dependências parcial etotal, respectivamente.

A análise bivariada de dependência nas AVD com variáveis sobre condições de saúde, sexo e idade revelou que estiveram estatisticamente associados: equilíbrio e mobilidade prejudicados $(\mathrm{RP}=2,14, \mathrm{IC} 95 \%=1,66-2,76)$, depressão ( $\mathrm{RP}=$ $1,88$, IC $95 \%=1,44-2,45)$, osteoporose ( $R P=1,85$, IC $95 \%=1,39-2,46)$, possuir 80 anos ou mais de idade $(\mathrm{RP}=1,69$, IC $95 \%=1,24-2,29)$, acuidade visual comprometida ( $\mathrm{RP}=1,62$, IC $95 \%=1,15$ $2,29)$, presença de doença $(R P=1,57, I C 95 \%=$ $1,10-2,24)$, sexo feminino ( $R P=1,55$, IC $95 \%=1,15$ $2,1)$, queda ( $R P=1,47$, IC $95 \%=1,12-1,93)$, déficit cognitivo ( $R P=1,46$, IC $95 \%=1,11-1,92$ ) e acuidade visual ruim ( $R P=1,33, I C 95 \%=1,01-1,75)$.

Os fatores associados estatisticamente significantes para dependência nas AIVD com variáveis sobre as condições de saúde, sexo e idade foram: déficit cognitivo ( $R P=1,76$, IC $95 \%=1,54$ 2,01 ), idade $\geq 80$ anos ( $R P=1,66$, IC $95 \%=1,47$ $1,88)$, equilíbrio emobilidade prejudicados ( $R P=$ $1,60$, IC $95 \%=1,40-1,83)$, doença pulmonar obstrutiva crônica ( $R P=1,47, I C 95 \%=1,20-1,80$ ), presença de doenças ( $R P=1,23, I C 95 \%=1,01$ $1,49)$, depressão ( $R P=1,21$, IC $95 \%=1,03-1,42$ ), hipertensão arterial $(R P=1,19, I C \quad 95 \%=1,01$ $1,40)$, ter idade entre 70 e 79 anos ( $R P=1,23, I C$ $95 \%=1,05-1,44)$ e sexo feminino $(R P=1,19, I C$ $95 \%=1,01-1,42$ ).

Na Tabela 3, observam-se a análise estratificada para a depen dência de cada AVD eas variáveis relativas às condições de saúde, sexo eidade. Entre as variáveis com maior número de associações com as AVD, destacam-se: equilíbrio emobilidade prejudicados, depressão, déficit cognitivo e idade $\geq 80$ anos.

$\mathrm{Na}$ análise estratificada para a dependência dasAIVD, como se observa na Tabela 4, ter idade $\geq 80$ anos, depressão, déficit cognitivo, equilíbrio e mobilidade prejudicados estiveram associados para todas as AIVD. Idade de 70 a 79 anos teve associação com todas as AIVD, exceto preparar refeições e realizar tarefas domésticas. 0 sexo feminino esteve associado à dependência ( total ou parcial) para realizar tarefas domésticas, manusear dinheiro, fazer compras e utilizar meio de transporte. As demais variáveis estudadas apresentaram número menor de associações.
Tabela 2. Condições de saúde e capacidade funcional dos idosos atendidos por equipes de Saúde da Família do Distrito Sanitário Leste. Goiânia (GO), 2001-2005 ( $n=388)$.

\begin{tabular}{|c|c|c|}
\hline Características & $n$ & $\%$ \\
\hline Acuidade visual ruim & & \\
\hline $\mathrm{Sim}$ & 265 & $\begin{array}{l}68,3 \\
317\end{array}$ \\
\hline
\end{tabular}

Acuidade auditiva ruim $\quad 123 \quad 31$

$$
\text { Sim }
$$$$
\text { Não } \quad 26969,3
$$

D epressão

Sim

$136 \quad 35,1$

$\mathrm{N}$ ão

25264,9

Déficit cognitivo

Sim $\quad 104 \quad 26,8$

Não $284 \quad 73,2$

Equilíbrio e mobilidade prejudicados

Sim

Quedas

Sim

Não

Presença de doenças

Sim

Não

H ipertensão

Sim

Não

Diabetes

Sim

Não

Osteoporose

Sim

Não

Problema cardíaco

Sim

Não

Artrite, artrose ou reumatismo

Sim

Não

Doença pulmonar obstrutiva crônica

Sim

Não

Acidente vascular cerebral

Sim

Não

Câncer

Sim

Não

Atividades de vida diária (AVD)

Independente

Dependência parcial

Dependência total

Atividades instrumentais de vida

diária (AIVD)

Independente

Dependência parcial

Dependência total
$296 \quad 76,3$

15038,7

23861,3

27570,9

11329,1

19851,0

19049,0

$\begin{array}{ll}59 & 15,2\end{array}$

$329 \quad 84,8$

$\begin{array}{ll}50 & 12,9\end{array}$

$338 \quad 87,1$

$37 \quad 9,5$

35190,5

$39 \quad 10,1$

34989,9

$16 \quad 4,1$

$6 \quad 1,5$

38298,5

$4 \quad 1,0$

38499,0

$253 \quad 65,2$

$123 \quad 31,7$

$12 \quad 3,1$

$153 \quad 39,4$

$177 \quad 45,7$

5814,9
37295,9 


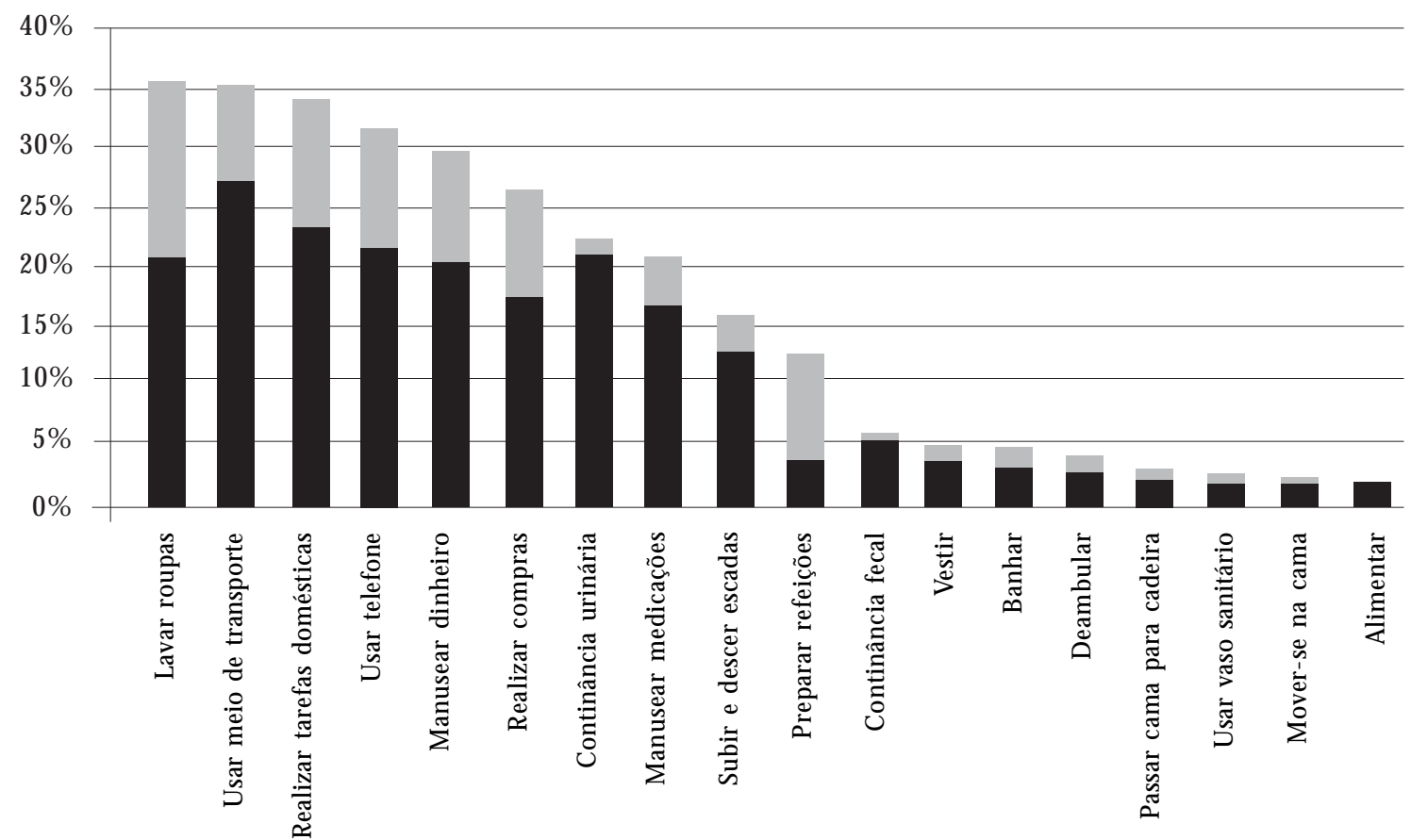

Nível de dependência
Parcial Total

Gráfico 1. Prevalência do nível de dependência parcial e total para todas as atividades de vida diária (AVD) e atividades instrumentais de vida diária (AIVD), em idosos atendidos pelas equipes da Saúde da Família. Distrito Sanitário Leste. Goiânia (GO), 2001-2005 (n=388).

\section{Discussão}

O perfil socioeconômico edemográfico dos idosos pesquisados é semel hante ao encontrado em outros estudos realizados em São Paulo, com amostra da população idosa domiciliar ${ }^{18 ;}$ em Porto Alegre e Aparecida de Goiânia, em áreas cobertas por equipes de Saúde da Família ${ }^{19,20}$, evidenciando uma população de idosos jovens, católicos, de baixa escolaridade erenda, aposentados, vivendo em domicílios multigeracionais.

Entre os idosos participantes deste estudo, a maioria era migrante. A cidade de Goiânia constitui há décadas centro de atração para migrantes, desde sua fundação, em 1960.

Em Goiânia, a ESF foi implantada prioritariamente em locais onde a população possuía baixo nível econômico enão tinha acesso a nenhum serviço na área da saúde ${ }^{11}$. D essa forma, é esperada a baixa renda dos gerontes participantes. 0 baixo grau de instrução dessa população é explicado pela infância e adolescência na zona rural, nos estados de origem, onde o acesso à escola era restrito ${ }^{3}$.

O fenômeno de os idosos residirem em domicílios multigeracionais, constituindo a principal fonte de suporte para a família, tem sido descrito como uma tendência na população mais pobre, em países em desenvolvimento ${ }^{21}$.

Apesar de os idosos possuírem baixa renda, a maioria tem moradia própria, pois se trata de uma região invadida pelos moradores e, posteriormente, legalizada pel os órgãos municipais.

As equipes de Saúde da Família precisam levar esse panorama em conta. Essa população de idosos jovens demanda ações de promoção de 
Tabela 3. Fatores associados à dependência para cada atividade de vida diária (AVD), em idosos atendidos por equipes da Saúde da Família. Distrito Sanitário Leste. Goiânia (GO), 2001-2005 (n=135).

\begin{tabular}{|c|c|c|c|c|c|}
\hline \multirow[b]{2}{*}{ Variáveis } & \multicolumn{5}{|c|}{ AVD } \\
\hline & Alimentar-se & Banhar-se & Vestir-se & $\begin{array}{c}\text { Usar } \\
\text { sanitário }\end{array}$ & $\begin{array}{c}\text { Passar de cama } \\
\text { para cadeira }\end{array}$ \\
\hline Sexo feminino & $0,481^{*}$ & 0,234 & 0,689 & $0,091^{*}$ & $0,450 *$ \\
\hline Idade $\geq 80$ anos & $0,024 *$ & $0,032 *$ & $<0,001^{*}$ & $0,057^{*}$ & $0,014 *$ \\
\hline Acuidade visual ruim & $0,382 *$ & $0,272^{*}$ & $0,224^{*}$ & $0,220 *$ & $0,164 *$ \\
\hline Acuidade auditiva ruim & $0,595^{*}$ & 0,785 & 0,549 & $0,239 *$ & $0,556 *$ \\
\hline Depressão & $0,022 *$ & $<0,001$ & 0,032 & $0,024 *$ & $0,051^{*}$ \\
\hline Déficit cognitivo & $0,006^{*}$ & $<0,001^{*}$ & $<0,001$ & $<0,001^{*}$ & $0,013^{*}$ \\
\hline Equilíbrio e mobilidade prejudicados & 0,003* & $<0,001 *$ & $<0,001^{*}$ & $<0,001^{*}$ & $<0,001^{*}$ \\
\hline Queda & $0,427 *$ & 0,131 & 0,199 & $0,151^{*}$ & 0,082 \\
\hline Presença de doenças & $0,437 *$ & $0,002 *$ & $0,050 *$ & $0,061^{*}$ & $0,208 *$ \\
\hline \multirow[t]{3}{*}{ Osteoporose } & $0,174^{*}$ & $<0,001$ & $0,218 *$ & $0,070 *$ & $0,326^{*}$ \\
\hline & \multicolumn{5}{|c|}{ AVD } \\
\hline & Deambular & $\begin{array}{c}\text { Subir e descer } \\
\text { escadas }\end{array}$ & $\begin{array}{l}\text { Mover-se I } \\
\text { na cama }\end{array}$ & $\begin{array}{c}\text { ncontinência } \\
\text { urinária }\end{array}$ & $\begin{array}{l}\text { a Incontinência } \\
\text { fecal }\end{array}$ \\
\hline Sexo feminino & $0,072^{*}$ & 0,033 & $0,518^{*}$ & 0,033 & $0,013^{* *}$ \\
\hline Idade $\geq 80$ anos & $<0,001$ & $<0,001$ & $0,151^{*}$ & 0,096 & $0,396 *$ \\
\hline Acuidade visual ruim & $0,024 *$ & 0,022 & $0,617^{*}$ & 0,033 & 0,604 \\
\hline Acuidade auditiva ruim & 0,424 & $<0,001$ & $0,267 *$ & 0,655 & 0,267 \\
\hline Depressão & 0,008 & $<0,001$ & $0,116^{*}$ & 0,002 & 0,008 \\
\hline Déficit cognitivo & 0,017 & $<0,001$ & $0,197 *$ & 0,391 & 0,960 \\
\hline Equilíbrio e mobilidade prejudicados & $<0,001 *$ & $<0,001$ & $0,003 *$ & 0,004 & 0,167 \\
\hline Queda & $0,083^{*}$ & 0,086 & $0,159 *$ & 0,059 & 0,424 \\
\hline Presença de doenças & $0,038 *$ & 0,216 & $0,437 *$ & 0,008 & 0,201 \\
\hline Osteoporose & $<0,001$ & $<0,001$ & $0,174 *$ & $<0,001$ & $0,453^{*}$ \\
\hline
\end{tabular}

* Teste Exato de Fisher; ** Variável associada estatisticamente $(I C<1,00)$.

saúde e prevenção de agravos, que deverão considerar tratar-se de uma população de baixa renda e escolaridade. As ações educativas precisarão estar adequadas ao nível de instrução e ao universo cultural desse grupo. Por sua vez, os domicílios multigeracionais podem representar a oportunidade de abordar as famílias de modo ampliado, ou seja, várias gerações ao mesmo tempo, envolvendo cada segmento na ação de cuidado intermembros do grupo familiar.

A avaliação da capacidade funcional é fundamental para determinar o comprometimento ea necessidade de auxílio para as atividades de manutenção e promoção da própria saúde e de gestão do ambiente domiciliar por parte dos idosos, podendo guiar as políticas públicas de atenção à saúde e as políticas sociais para este segmento. Em outros estudos ${ }^{22,23}$, o percentual deidososque necessitavam de ajuda para executar as AVD e AIVD foi semelhante ao encontrado na presente pesquisa, 35,8\% e $60,6 \%$, respectivamente.

A dependência para essas atividades gera a necessidade de apoio por parte de cuidadores, os quais, independentemente de serem formais ou informais, precisam de preparo e suporte adequados. Cabeà Estratégia Saúde da Família atender aos usuários em suas necessidades; assim, a responsabilidade do preparo e do apoio aos cuidadores recai sobre os profissionais que atuam na área. É importante conhecer quais são as atividades de maior depen dência para a elaboração de um plano de ação que integre atividades de promoção da saúde, de prevenção e tratamento desses comprometimentos.

Dentre as AVD, a incontinência urinária tem sido a mais prevalente ${ }^{6,21,24}$. $\mathrm{N}$ a presente investi- 
Tabela 4. Fatores associados à dependência para cada atividade instrumental de vida diária (AIVD), em idosos atendidos por equipes da Saúde da Família. Distrito Sanitário Leste. Goiânia (GO), 2001-2005 (n=235).

\begin{tabular}{|c|c|c|c|c|}
\hline \multirow[b]{2}{*}{ Variáveis } & \multicolumn{4}{|c|}{ AIVD } \\
\hline & $\begin{array}{l}\text { Preparar } \\
\text { refeições }\end{array}$ & $\begin{array}{c}\text { Realizar tarefas } \\
\text { domésticas }\end{array}$ & Lavar roupa & $\begin{array}{l}\text { M anusear } \\
\text { medicação }\end{array}$ \\
\hline $\begin{array}{l}\text { Sexo feminino } \\
\text { Idade }\end{array}$ & 0,198 & 0,044 & 0,984 & 0,962 \\
\hline 70 a 79 anos & 0,185 & 0,256 & 0,014 & $<0,001$ \\
\hline$\geq 80$ anos & $<0,001$ & $<0,001$ & $<0,001$ & $<0,001$ \\
\hline Depressão & $<0,001$ & 0,003 & 0,009 & $<0,001$ \\
\hline Déficit cognitivo & $<0,001$ & $<0,001$ & $<0,001$ & $<0,001$ \\
\hline Equilíbrio e mobilidade prejudicados & $<0,001$ & $<0,001$ & $<0,001$ & $<0,001$ \\
\hline Presença de doenças & 0,407 & $<0,001$ & 0,002 & 0,181 \\
\hline Hipertensão arterial & 0,881 & 0,007 & 0,107 & 0,773 \\
\hline \multirow[t]{2}{*}{ Doença pulmonar obstrutiva crônica } & $0,291 *$ & 0,062 & $<0,001$ & $0,448 *$ \\
\hline & \multicolumn{4}{|c|}{ AIVD } \\
\hline Variáveis & $\begin{array}{l}\text { Usar } \\
\text { telefone }\end{array}$ & $\begin{array}{l}\text { Manusear } \\
\text { dinheiro }\end{array}$ & Fazer compras & $\begin{array}{c}\text { Usar meio de } \\
\text { transporte }\end{array}$ \\
\hline Sexo feminino & 0,174 & 0,002 & $<0,001$ & $<0,001$ \\
\hline \multicolumn{5}{|l|}{ Idade } \\
\hline 70 a 79 anos & 0,006 & 0,018 & 0,012 & 0,003 \\
\hline$\geq 80$ anos & $<0,001$ & $<0,001$ & $<0,001$ & $<0,001$ \\
\hline Depressão & $<0,001$ & 0,001 & $<0,001$ & $<0,001$ \\
\hline Déficit cognitivo & $<0,001$ & $<0,001$ & $<0,001$ & $<0,001$ \\
\hline Equilíbrio e mobilidade prejudicados & $<0,001$ & $<0,001$ & $<0,001$ & $<0,001$ \\
\hline Presença de doenças & 0,687 & 0,066 & 0,004 & 0,051 \\
\hline Hipertensão arterial & 0,346 & 0,103 & 0,012 & 0,192 \\
\hline Doença pulmonar obstrutiva crônica & 0,086 & 0,206 & 0,118 & 0,442 \\
\hline
\end{tabular}

* Teste Exato de Fisher.

gação, esta atividade obteve maior comprometimento, principalmente a incontinência urinária ocasional, relatada pelas mulheres, relacionada ao uso de medicamentos (diuréticos). A literatu$\mathrm{r}^{25}$ apresenta a fraqueza do assoalho pélvico decorrente do envelhecimento e multiparidade como principais fatores que favorecem tal ocorrência. Essa condição pode afetar a vida social e as demandas de higiene.

Entre os participantes, a dificuldade de subir e descer escadas foi uma das atividades com maior prevalência. Esse comprometimento pode ser justificado pela deterioração progressiva das cartilagens, além da perda de tamanho, força, flexibilidade e resistência dos músculos, no processo de envel hecimento, eagravadas pelo sedentarismo ${ }^{26}$. Além disso, a capacidade de discernimento de um degrau e outro e a acuidade visual comprometida são algumas das queixas relatadas que impediam a realização dessa atividade.

As atividades como realizar tar efas domésticas, lavar roupas e preparar alimentos foram prevalentes entre os idosos dependentes. É importante salientar que, em algumas famílias, especialmente naquelas em que as filhas adultas ainda moram com os pais, há uma tendência de substituição deles nas atividades domésticas, tentando preservá-los de esforços físicos.

Os profissionais de saúde podem encorajar os idosos a permanecerem por mais tempo realizando atividades que demandem esforço físico, além de ajudar os familiares a compreenderem a necessidade de realização de movimentos corporais, como forma de manter habilidades como a marcha, a elevação dos membros inferiores para subir e descer degraus. Por sua vez, exercícios es- 
pecíficos devem ser ensinados, tais como aqueles indicados para o fortalecimento do assoal ho pélvico, para minimizar os quadros de incontinência urinária em mulheres.

As dificuldades de tomar medicação, usar 0 tel efone, manusear dinheiro, fazer compras eusar meio de transporte, segundo relatos dos idosos, tinham relação com a falta de escolaridade. Isso pode prejudicar a vida social do idoso e implica transtornos para ele e sua família, diminuindo sua autonomia.

As escalas de avaliação de Mahoney e Barthe ${ }^{14}$ e de Lawton et al. ${ }^{15}$ têm sido amplamente utilizadas em estudos dessa natureza. No entanto, os estudiosos têm utilizado esses instrumentos para análise num plano geral $6,20,23$, reportando-se ao nível de dependência. De modo geral, as pesquisas avaliam as tarefas em forma de blocos, 0 que dificulta 0 aprofundamento da questão $0^{27}$. N este estudo, para facilitar a compreensão dos fatores envolvidos no declínio funcional, estratificaram-se as variáveis para cada atividade, para que as estratégias possam ser desenvolvidas para cada situação, pois o tipo de ajuda necessária, as demandas de serviços e os custos decorrentes das incapacidades são muito diferentes.

Vários estudos descrevem como fatores associados à dependência para as AVD e AIVD: 0 sexo feminino, idade, equilíbrio e mobilidade prejudicados, presença de doenças ${ }^{6}$, depressãa $0^{6,21}$, visão comprometida ${ }^{6,26}$, déficit cognitivo ${ }^{21}$, quedas $^{21,26}$, dificuldade auditiva, hipertensão arterial e bronquite ${ }^{26}$.

N este estudo, houve prevalência das mulheres em algum tipo de dificuldade. Estudiosos ${ }^{22,28}$ afirmam que tal associação provavelmente deve-se ao fato de as mulheres possuírem maior expectativa devidae, consequentemente, maior risco dedesenvolverem doenças crônicas incapacitantes.

Os idosos pesquisados com faixa etária mais elevada apresentaram maior prevalência de dependência funcional. Em geral, limitações funcionais são mais frequentes em indivíduos mais idosos, pelo próprio declínio da função de alguns sistemas orgânicos, gerando risco de ter alguma restrição funcional ${ }^{29}$.

Estudos mostram que, durante o envel hecimento, os idosos queixam-se de dificuldade visual e auditiva ${ }^{6,20,23}$. Essas deficiências não impedem um envel hecimento bem-sucedido. Para isto, é necessário que adotem mecanismos adaptativos para minimizar as dificuldades. Órteses para correções podem permitir os ajustes dessas deficiências. Contudo, nessa fase da vida, a adaptação pode dificultar o uso das órteses. A maioria dos entrevistados relatou possuir óculos e não usá-los por incômodo ou falta decostume. A acuidade visual podeinterferir na realização dasAVD, como o uso de escadas, deambulação e na continência urinária, sendo decorrente da dificuldade de visualização dos obstáculos a serem percorridos, embora as maiores queixas tenham sido o equilíbrio emobilidade. 0 comprometimento da acuidadeauditiva esteveassociado estatisticamente com o subir e descer escada, contudo não se encontrou uma relação para tal associação.

0 processo cognitivo é a interação entre as funções psicológicas (motivação, atenção, percepção, aprendizagem, memória, raciocínio, juízo, imaginação, pensamento ediscurso) eo processo fisiológico que começa com a estimulação dos órgãos sensoriais (visão, tato, audição, paladar, olfato) ${ }^{25,30}$. 0 comprometimento das funções cognitivas, como déficit de memória de fixação e evocação, perturbação na orientação espaço-temporal, dificuldade de atenção, prejuízo na capacidade de julgamento, apraxias e afasias, implica perdas de independência e autonomia25.

0 déficit cognitivo apresentou associação para a maioria das AVD e todas as AIVD. Os entrevistados que apresentavam comprometimento das funções cognitivas queixaram-seprincipalmente de esquecimento ao cozer os alimentos, deixando-os queimar, e detomar medicação nos horários corretos.

A depressão éuma síndromemultifatorial que se caracteriza por um estado patológico de sofrimento psíquico com distúrbios afetivo e de humor $^{31}$. A maioria das depressões nos idosos é atípica, frequentemente associada à redução da autoestima, falta deânimo, sensação de vazio, sentimento de culpa, pessimismo, desamparo, inutilidade, irritabilidade, inquietação e fadiga ${ }^{25}$. Entre os entrevistados, a depressão foi considerada um fator de risco para dependência de todas as AIVD e para as AVD, exceto mover-se na cama.

A presença de sintomas depressivos traz consequências negativas para a qualidade de vida do indivíduo e grandes implicações sociais. As medidas de intervenções podem identificar as causas tratáveis desses transtornos, mantendo o indivíduo na comunidade pelo maior tempo possível e aproveitando ao máximo sua autonomia.

0 equilíbrio ea mobilidade prejudicados estiveram estatisticamente associados para quase todas as atividades, exceto para a incontinência fecal. Autores ${ }^{2,25}$ revelam que disfunções motoras, sensoriais, depressão, déficit cognitivo e medicamentos (benzodiazepínicos, antipsicóticos, antidepressivos e outros) ocasionam a instabilidade 
postural e a alteração da marcha, aumentando a possibilidade de risco para quedas que poderão prejudicar a execução das atividades cotidianas.

Uma medida importanteé prevenir os possíveis acidentes domésticos que envolvam quedas, retirando tapetes, objetos que dificultam a circulação e levam ao tropeço, colocando piso antiderrapante, principalmentenas escadas, eiluminação adequada em todos os ambientes. Além disso, as barrei ras arquitetônicas devem facilitar a locomoção do idoso. Por exemplo: as calçadas não devem ter vegetação abundante, pisos rachados e desnivelados; as escadas não podem ter uma altura que leve ao esforço exagerado para usá-la; o corrimão deve existir sempre em escadas e corredores.

Um dos desafios a serem enfrentados no processo do envelhecimento é a presença de dupla carga de doenças. A maioria dos participantes referiu ser portadora de uma ou mais enfermidades, principalmentehipertensão arterial ediabetes. Essa prevalência também foi observada em outros estudos $5^{5,19,21}$ e identificada sua influência para a dependência nas AIVD e AVD ${ }^{32}$.

A osteoporose é uma síndrome caracterizada pela redução ou deterioração do tecido ósseo, levando à fragilidadeóssea, tendo como um dos sintomas a dor ${ }^{25}$. Este sintoma pode contribuir para a limitação do idoso em realizar atividades como banhar-se, deambular, subir ou descer escadas. A associação entre a osteoporose e a incontinência urinária ocasional pode estar relacionada a limitações no movimento, em decorrência da dor.

A doença pulmonar obstrutiva crônica (DPOC) aumenta a resistência das vias aéreas, gerando a perda da capacidade de retração elástica dos pulmões e a diminuição da oferta de oxigênio, sendo a dispneia um dos sintomas ${ }^{33}$. A dependência para a atividade de lavar roupa teve forte associação com essa doença. Trata-se de tarefa que exige esforço físico, que aumenta a demanda de oxigênio, levando ao cansaço edispneia para os idosos com esse tipo de problema desaúde, impedindo-os derealizá-la. Alguns equipamentos facilitadores, como máquinas delavar roupas, são de grande ajuda para compensar a dificuldade na realização dessa atividade.

A hipertensão arterial pode ser explicada pe las alterações fisiológicas, como progressivo aumento da rigidez da árvore arterial, ocasionando redução da distensibilidade vascular e levando ao contínuo aumento da pressão arterial ${ }^{34}$. A diminuição do reflexo do desempenho do coração e a dificuldade de aumentar o débito cardíaco têm como consequência a queda do consumo máximo de oxigênio, por isso os idosos apresentam dificuldades em realizar tarefas que demandem maior esforço físico, como atividades domésticas e fazer compras.

A Organização Mundial da Saúdelançou políticas do envelhecimento ativo que visam otimizar a saúde, a participação ea segurança, de modo a melhorar a qualidade de vida à medida que as pessoas envelhecem ${ }^{35}$. Para minimizar/prevenir as consequências da senilidade, é necessário que os profissionais de saúde pública estabeleçam prioridades de atuação. A detecção de novos casos e o acompanhamento dos portadores de enfermidades poderão prevenir econtrolar os agravos, enquanto medidas de promoção de saúde devem contar com a participação da comunidade na busca de um envelhecimento ativo, que é uma meta importante para prevenir os fatores de risco que aceleram o processo incapacitante.

0 atendimento integral em saúde prevêuma ação intersetorial, com atividades multiprofissionais tais como inclusão digital, estimulação cognitiva, exercícios físicos para a terceira idade, que contribuam para a qualidade de vida dessa população.

Dentre as limitações do estudo, destaca-se 0 intrínseco ao delineamento transversal, o qual impossibilita verificar temporalidade das associações encontradas. 0 processo amostral incluiu somente os idosos do DSL, não sendo, assim, uma amostra representativa do município de Goiânia. Porém, pressupõe-se que represente os idosos atendidos pelas equipes de Saúde da Família, pois os bairros que possuem a ESF implantados são demográfica eeconomicamentesemelhantes. Sugere-sea realização de estudos multicêntricos, que incluam amostras representativas de idosos dos municípios, a fim de se conhecerem as condições de saúde e capacidade funcional de idosos de diferentes classes sociais. A realização de estudos de coorte também é importante, tendo em vista quehá pou cos estudos Iongitudinais sobre a capacidade funcional.

0 conjunto deinstrumentos deavaliação utilizados prevê a participação dos sujeitos de forma ativa, isto é, eles devem ser capazes de responder às questões, como no caso do exame do estado mental, ou do relato de capacidade deatividades de vida diária. Outras avaliações ficam comprometidas diante de condição de acamado. Assim, idosos com comprometimento da condição de saúde, com dificuldade de comunicação, acamados, não podem ser avaliados no conjunto com essas escalas. Neste estudo, 11 pessoas estavam nessas condições $(2,7 \%)$. 
Diante do grande número de desfechos e variáveis estudadas, não foi realizada no presente estudo análise multivariada, o que deve ser feito em estudos futuros.

Os dados identificados por esta pesquisa são importantes para seconhecer a situação dosidosos de baixa renda, atendidos pelo sistema público de saúde. Podem auxiliar no planejamento das ações de saúde, de acordo com os fundamentos do SUS. Além disso, apontam perspectivas para estudos futuros, em quea análi isefuncional dos idosos leve em conta os vários domínios de desempenho.

Na promoção da saúde, fica evidente o desafio para se desenvolverem estratégias capazes de estimular a população a adotar estilo de vida saudável, em especial a prática de atividades físicas, que desempenham papel fundamental também na recuperação da capacidade funcional ${ }^{36}$.

Planejar tais estratégias demanda atuação eficaz da equipe multidisciplinar, com visão ampla da clínica gerontológica e geriátrica, tomando como base 0 atendimento humanizado e integrado aos princípios da Atenção Básica.

Em síntese, os idosos apresentaram, na maioria, idade entre 60 e 69 anos, com predomínio do sexo feminino $(58,5 \%)$, renda menor ou igual a dois salários (77,3\%), analfabetos (39,7\%), relatando uma ou mais doenças crônicas não transmissíveis (70,9\%), em especial hipertensão arterial $(51 \%)$ e diabetes (15,2\%). A presentaram dependência (parcial ou total) para as AVD de $34,8 \%$ e de $60,6 \%$ para as AIVD. Os fatores mais frequentemente associados às dependências $(p \leq$ $0,05)$, tanto para AVD como para as AIVD, foram: equilíbrio e mobilidade prejudicados, de pressão, déficit cognitivo, idade $\geq 80$ anos.

\section{Referências}

1. Kalache A. O mundo envelhece: é imperativo criar um pacto de solidariedade social. Cien Saude Colet 2008; 13(4):1107-1111.

2. Brasil. Ministério da Saúde. Departamento de Atenção Básica. Envelhecimento e saúde da pessoa idosa. Brasília: Ministério da Saúde; 2006.

3. Brasil. Instituto Brasileiro de Geografia e Estatística. Perfil dos idosos responsáveis pelos domicílios no Brasil 2000. Rio de Janeiro: Ministério do Planejamento; 2002.

4. Lima-Costa M F, Barreto S, Giatti L, U choa E. Desigualdade social e saúde entre idosos brasileiros: um estudo baseado na Pesquisa Nacional por Amostra de Domicílios. Cad Saude Publica 2003; 19(3):745757.
Conclui-se que a maioria dos idosos participantes apresentou envelhecimento malsucedido, caracterizado por comorbidades edependência para realizar as atividades diárias. Recomendam-se investigações longitudinais dos fatores de risco para dependência duranteo envelhecimento, demodo a facilitar o planejamento de estratégias de promoção de saúde e de prevenção das incapacidades.

\section{Colaboradores}

DP N unes participou da coleta, do processamento, análise einterpretação de dados e redação do artigo. AYK Nakatani coordenou o trabalho de campo do estudo, participou da elaboração dos instrumentos da coleta de dados, análise de dados, interpretação e redação final do artigo. EA Silveira e M M Bachion participaram da análisee interpretação dos dados, revisão de aspectos metodológicos e redação final do artigo. M R Souza participou da interpretação e da redação final do artigo.

\section{Agradecimentos}

Ao Conselho Nacional de Desenvolvimento $\mathrm{Ci}$ entífico e Tecnológico (CN Pq). A todos os idosos que participaram do estudo. Aos profissionais das equi pes de saúde do DSL, principalmente os agentes comunitários, pela disponibilidade e paciência durante a busca ativa dos participantes. Aos colaboradores da coleta de dados: TM Silvério, DM Jesus, EC Costa, LB Silva, LF Santos, RF M arçal.
5. Lima-Costa M F, Loyola Filho Al, M atos D. Tendências nas condições de saúde e uso de serviços de saúde entre idosos brasileiros: um estudo baseado na Pesquisa Nacional por Amostra de Domicílios (1998, 2003). Cad Saude Publica 2007; 23(10):2467-2478.

6. Nakatani AYK, Costa EFA, Teles SA, Silva LB, Rego $M A B$, Souza ACS et al. Perfil sociodemográfico e avaliação funcional de idosos atendidos por uma equipe de saúde da família na periferia de Goiânia, Goiás. Rev Soc Bra Clín M éd 2003; 1(5):131-136.

7. Veras R. Modelos contemporâneos no cuidado à saúde. Revista USP 2001; 51:72-85. 
8. Costa EFA, Porto CC, Almeida JC, Cipullo JP, Martin JFV. Semiologia do idoso. In: Porto CC. Semiologia médica. 5a ed. Rio de Janeiro: Guanabara Koogan; 2005. p. 154-183.

9. Fried LP, Ferrucci L, Darer J, Williamson JD, Anderson G. Untangling the concepts of disability, frailty and comorbidity: implications for improved targeting and care. J Gerontol 2004; 59(3):255-263.

10. Silveira EA, Peixoto MRG, Sousa LM, Costa R, Assis VC. Indicadores de saúde infantil em Goiânia, Goiás, no período de 2000 a 2004, segundo dados do Sistema de Informação da Atenção Básica (SIAB). Revista Eletrônica de Enfermagem [periódico na Internet]. 2007 set-dez [acessado 2008 out 03] 9(3):674-686. Disponível em: http://www.fen.ufg.br/ revista/v9/n3/v9n3a08.htm

11. Brasil. M inistério da Saúde. Departamento de Atenção Básica. Política nacional de atenção básica. Brasília: M inistério da Saúde; 2006.

12. Almeida OP. M iniexame do estado mental e o diagnóstico de demência no Brasil. Arq Neuropsiquiatr 1998; 56(3B):605-612.

13. Almeida OP, Almeida SA. Confiabilidade da versão brasileira da escala de depressão em geriatria (GDS) versão reduzida. Arq N europsiquiatr 1999; 57(2B):421-426.

14. Mahoney FI, Barthel DW. Functional evaluation: the Barthel ADL index. M d M ed J 1965; 14:61-65.

15. Lawton M P, Moss M, Fulcomer M, Kleban MH. A research and service-oriented multilevel assessment instrument. J Gerontol 1982; 37:91-99.

16. Laks J, Batista EMR, Guilherme ERL, Contino ALB, Faria MEV, Rodrigues CS et al. Prevalence of cognitive and functional impairment in communitydwelling elderly: importance of evaluating activities of daily living. Arq Neuropsiquiatr 2005; 63(2a):207-212.

17. Brasil. Conselho Nacional de Saúde. Resolução 196/ 96 - sobre pesquisa envolvendo seres humanos. Bioética 1996; 4(2):15-25.

18. Lebrao ML, Laurenti R. Saúde, bem-estar e enveIhecimento: o estudo SABE no município de São Paulo. Rev Bras. Epidemiol. 2005; 8(2):127-141.

19. Souza LM, Morais EP, Barth QEM . Características demográficas, socioeconômicas e situação de saúde de idosos de um Programa de Saúde da Família de Porto Alegre, Brasil. Rev Lat Am Enfermagem [periódico na Internet] 2006 nov-dez [acessado 2007 jul 24]; 14(6): [on line]. Disponível em: http:// www.eerp.usp.br/rlae

20. Araujo MAS, Nakatani AYK, Silva LB, Bachion M M . Perfil do idoso atendido por um Programa de Saúde da Família em Aparecida de Goiânia (GO). Revista da UFG [periódico na Internet] 2003 dez [acessado 2007 jan 30]; 5(2): [on line]. Disponível em: http://www.proec.ufg.br/revista_ufg/idoso/ perfil.html

21. Ramos LR. Fatores determinantes do envelhecimento saudável em idosos residentes em centro urbano: Projeto Epidoso, São Paulo. Cad Saude Publica 2003; 19(3):793-797.

22. Santos KA, Koszuoski R, Dias-da-Costa JS, Pattussi M P. Fatores associados com a incapacidade funcional em idosos do município de Guatambu, Santa Catarina, Brasil. Cad Saude Publica 2007; 23(11):27812788.
23. Feliciano AB, Moraes AS, Freitas ICM . 0 perfil do idoso de baixa renda no município de São Carlos, São Paulo, Brasil: um estudo epidemiológico. Cad Saude Puública 2004; 20(6):1575-1585.

24. Maciel AC. Sinais e sintomas em urologia geriátrica. In: Guimarães RM, Cunha UG. Sinais e sintomas em geriatria. 2ª ed. São Paulo: Atheneu; 2004. p. 219-227.

25. Vieira EB. M anual de gerontologia: um guia teóricoprático para profissionais, cuidadores e familiares. Rio de Janeiro: Revinter; 2004.

26. Rosa TEC, Benício MHA, Latorre MRDO, Ramos $L R$. Fatores determinantes da capacidade funcional entre idosos. Rev Saude Publica 2003; 37(1):40-48.

27. Barbosa $A R$, Souza JMP, Lebrão R, Laurenti $R$, $M$ arucci M F. Functional limitations of Brazilian elderly by age and gender differences: data from SABE survey. Cad Saude Publica 2005; 21(4):1177-1185.

28. Costa M FL, Firmo JOA, U choa E. Differences in self-rated health among older adults according to socioeconomic circumstances: the Bambui $\mathrm{H}$ ealth and aging study. Cad Saude Publica 2005; 21:830-839.

29. Maciel ACC, Guerra RO. Influência dos fatores biopsicossociais sobre a capacidade funcional de idosos residentes no Nordeste do Brasil. Rev Bras Epidemiol 2007; 10(2):178-189.

30. M achado JC, Ribeiro RC, Leal PFG, Cotta RMM . Avaliação do declínio cognitivo e sua relação com as características socioeconômicas dos idosos em Viçosa (M G). Rev Bras Epidemiol 2007; 10(4):592-605.

31. Irigaray TQ, Schneider RH. Prevalência de depressão em idosas participantes da Universidade para a Terceira Idade. Rev Psiquiatr 2007; 29(1):19-27.

32. Alves LC, Leimann BCQ, Vasconcelos M EL, CarvaIho MS, Vasconcelos AGG, Fonseca TCO, Lebrão $M L$, Laurenti R. A influência das doenças crônicas na capacidade funcional dos idosos do município de São Paulo, Brasil. Cad Saude Publica 2007; 23(8):1924-1930

33. Francisco PMSB, Donalisio MR, Barros MBA, César CLG, Carandina L, Goldbaum M. Fatores associados à doença pulmonar em idosos. Rev Saude Publica 2006; 40(3):428-435.

34. Liberman A, Liberman M, Saraiva JFK. Envelhecimento do sistema cardiovascular do fisiológico ao patológico. In: Liberman A, Freitas EV, Savioli Neto $F$, Taddei CFG. Diagnóstico e tratamento em cardiologia geriátrica. Barueri (SP): M anole; 2005. p. 5-12.

35. Organização Mundial da Saúde (OMS). Envelhecimento ativo: uma política de saúde. Brasília: Organização Pan-Americana da Saúde; 2005.

36. Benedetti TRB, Gonçalves HT, Mota JAPS. Uma proposta de política pública de atividade física para idosos. Texto \& Contexto Enfermagem 2007; 16(3):387-398.

Artigo apresentado em 10/07/2008

Aprovado em 04/09/2008

Versão final apresentada em 30/10/2008 